\title{
Analyzing Optimization Strategies and Techniques for Study of Power Management in Embedded System
}

\author{
Sadaf Qasim \\ Department of Electrical and Electronics, SRMS, College of Engineering \& Technology Bareilly, UP, India
}

Email address:

Sadaf.riya@gmail.com

\section{To cite this article:}

Sadaf Qasim. Analyzing Optimization Strategies and Techniques for Study of Power Management in Embedded System. International Journal of Industrial and Manufacturing Systems Engineering. Vol. 6, No. 2, 2021, pp. 35-38. doi: 10.11648/j.ijimse.20210602.12

Received: June 5, 2021; Accepted: July 5, 2021; Published: July 15, 2021

\begin{abstract}
Designing of Power Management requires the reduction of power dissipation in every section and unit of design system. During all these steps of designing process, it incorporates the constraints within the system and its performance. An embedded system inculcates rigid constraints to power consumption and memory of suitable size along with other terms like weight and cost. The basic Power consumption through embedded system based devices is a complex issue. The system always has to extend its battery or may reduce the environmental issues. Basically, it is a pure hardware issue. In latest development, an embedded system within its upgraded technology takes responsibility for all the power management. Embedded systems can be said to come into existence for specific purposes and should be optimized in order to meet all sorts of constraints. By constraint, it is meant to be system performance, timing, power and cost. The designing process of such system therefore involves many optimization techniques. In this paper, we will study and analyze the techniques, strategies, main advantages and disadvantages of applying optimization method of constraint problem in designing of embedded system. Also this will reviews the procedure of characterizing power management in minimizing power consumption.
\end{abstract}

Keywords: Optimization Techniques, Embedded System, Power Management, Power Consumption

\section{Introduction}

The system may be defined as set of several units that are useful to serve any purpose. And System design may be term as the process of implementing any particular section in response to optimization. Example for this may be considered as follows, suppose, if a upmarket personal computer has to be monitored and optimized any clock pulse at its maximum range, whereas an electronic system which is operated by any battery or some wireless connection may be optimized to some minimum dissipation of power for better performance [9]. If we say, an embedded system that is a group of hardware, and software will executes system which is connected to peripheral for the application through different software. For examples, let us take any peripheral devices that include unit to display, LAN network, and input device such as keyboard [6].

Power efficiency is important factor in any management unit that is needed to be obtained by reduction in dissipation factors in every section of system design. If we reduce the power intake of every single unit and constituents the hardware base that can minimize its dissipation factor of power [10]. Therefore we can see that the peripheral unit always tends to consume minute significant figures from the total system of power.

The main role or key notes of embedded based system which may be define as both hardware and software designing process combination. This hardware and software combine designing can be term as the process of setting state of defining any hardware as well as some portion of software into any embedded system though we know the dependencies of two with each other [3]. In designing, the manufacturer can rely on their experience where that have seen the past systems of estimating the resource which will ultimately tend to gives the need of new system. There are plenty of research that is taking places in theses area of hardware and software combine designing which has full focused to make the process simple, less complex yet accurate.

\section{Embedded Systems Design}

Basically all the element of typically embedded system is 
implemented on one single chip that constitute a device what we call it as system on-chip as we can see the description in Figure 1. An embedded system holds explicit characteristics which make it distinguish in all other computing devices [4, 7].

Some basic characteristics may be term as follow:

Single function: It performs any specific task repeatedly make it distinguish.

Real-time operation: If we see accuracy, we cannot ignore time constraint which is very important factor in executing job on any embedded based systems. Therefore even define portion of executing hinder can lead to malfunction of whole system in the end.

Rigid constraints: So because of the characteristic of embedded based systems design, their functioning with respect to size, performance and working adopt rigid constraint so as to have fast and quick response [8].

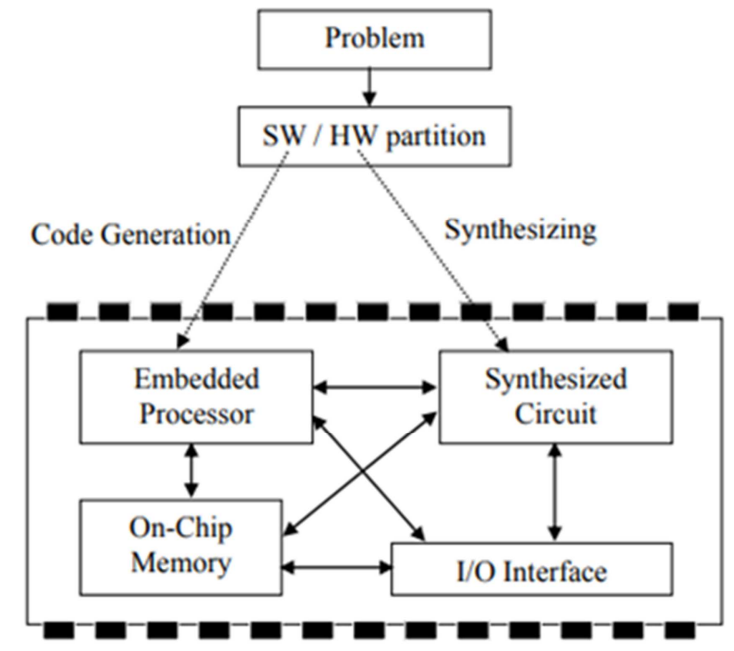

Figure 1. Embedded System Structure.

\section{Literature Analysis}

\subsection{Power Compiling Software}

Analysis of power of different adeptness of it have introduce in designing embedded based operating system so to have characterization of instruction on level and simulation that is elementary hardware. Some techniques need to revise to improve and to have efficiency which is up to mark of software analysis with some statistical review that has been proposed [14]. The design paradigm should have proper research in evaluate the designing process of consumption of power in reference to hardware and software based system. Optimizations are done for embedded software which consumes power through compiler which has checked to do optimizations and memory management is being introduced for record purpose. Previously we have awareness of compilation of techniques which was limited.

\subsection{Dynamically Management of Power}

Dynamic Management of Power may refers to select the priority to $\mathrm{ON}$ and $\mathrm{OFF}$ any system components which are idle or in working occasionally, shows particularly the importance of effective technique to reduce power dissipation in all these systems. To inculcates and adopt dynamic management scheme of power, we need design of some preexisting complex system which is again difficult process and includes many iterations. We need a careful debugging of process to validate our design process [11]. The main aim of management policy which dynamically able to minimize the consumption in power and to do so we have to employ electronic system integrant of different states, where each system represents some accomplishment and different level of consumption which include power utility.

For constructing and managing a new kind of problem based on constrained to optimize the admissible power electronic systems in order to minimize dissipation factor or power being monitored under constraint. This may be term as optimization problem policy. In such proposal of model, there generates a precise mathematical formulation which defines problem at the same time try to model and present a process their solution. And finally those solutions which has been computed to solve by a linear optimization problem [9, 11]. This viewpoint which has been based on power devices managed and the caseloads of optimization based techniques will now be based on discrete-time theory which was given by Markov decision processes.

The main object of this function is to expect system performance which includes all the related waiting time and different jobs and then its constraint which is expected to consumer's power transfer purpose from one state server to others state server for next cycle.

\subsection{Power Management Software}

For minimizing power consumption during its usage, software can take several measures to serve the purpose. They are: Peripherals devices should be turn OFF when they not in working state.

We can adjust their system frequency as well as voltage according requirement of current by system. They are term as Dynamic Voltage and Frequency Scaling (see Figure 2).

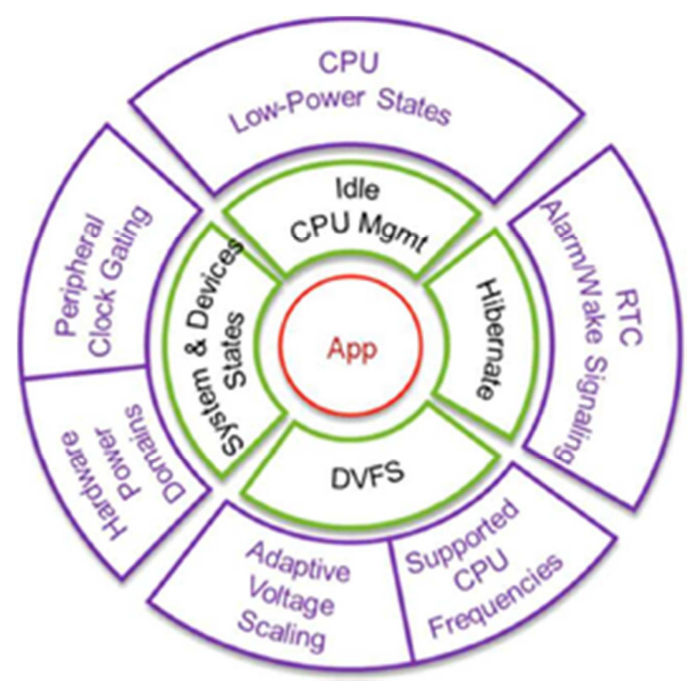

Figure 2. Embedded System Structure. 


\subsection{Operating Points and their Cases Involve}

We know that RTOS will not able to optimize power, as they are not aware of the system performance whose requirements for the developing code and their execution of system performance readily is the primary source which has influences energy consumption [12].

So commonly we can approach the developer in order to study and do analysis of application that define a set of number which are term as "use cases" and are included with some specific situations in which these device are used leading to one of a kind of state [15].

Any measuring device with handheld can have certain cases such as:

Measurement

Data Displaying

Data Uploading

Idle cases

\section{Strategies of Power Optimization}

The system architecture is generally use with static as well as dynamic management to have power mechanisms that helps us to manage consumption of different power unit [11]. So, a sorted discussion of power management policy can be define as the main strategy in developing and determining whether we have to perform certain management in context to power operations and working [10]. In short management policy generally examines different aspect of system which determines its action that has to be taken.

And due to the symptoms of power up and down, there can be different modes which should be examined carefully. Thus we have a mode of several fluctuations which determines which switch is to be chosen and what has to be puts under analysis of system activity. Therefore following issues which come into action:

Unnecessary Power causes power down issue which is needed to be avoided.

Penalties in performance due to power down repeatedly lead to loses severely.

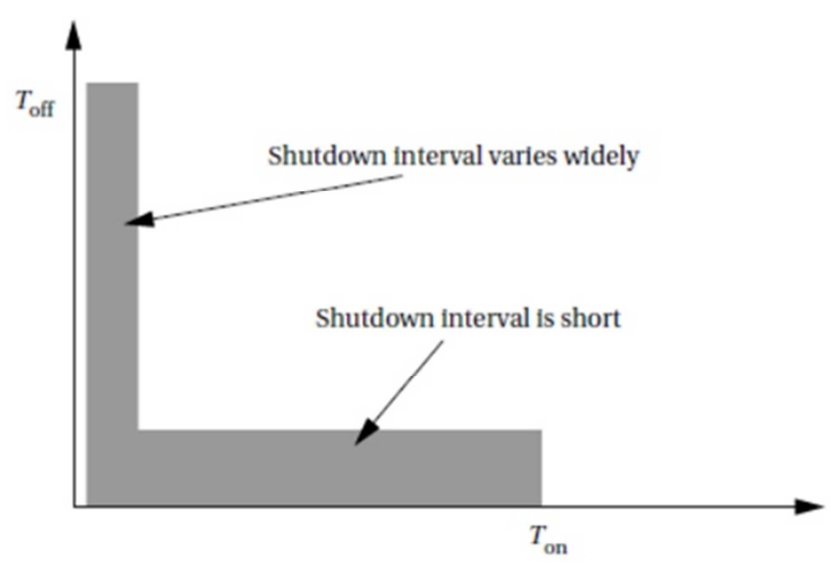

Figure 3. L-Distribution usage.

The different choices to keep $\mathrm{T}_{\text {off }}$ and $\mathrm{T}_{\text {on }}$ must be determined by some analyses of experimentation [15]. The plot can be observe which shows the idle time $\left(\mathrm{T}_{\text {off }}\right)$ which have graphics terminal versus the preceding immediately ON time $\left(\mathrm{T}_{\mathrm{on}}\right)$. The result can be evaluated with the help of an L-distribution process illustrated in Figure 3. Here in this distribution process, the left over period where we have idle case came in to existence after suitable duration of active (ON) period that is kept usually for short span; however the length of left over duration or idle case can be seen after a short ON time which is uniformly distributed.

\section{Optimization of Power}

If we talk of synthesis level of high magnitude of level (also term as HLS), it can be define as the process of plotting or describing the behavioral specification in any specific system where we have description to transfer the register [16]. And the outcome his synthesis is so conceptual that a systematic view of any data or logical aspect within this can be said to have control unit. High-level synthesis have different task but main can be classified as: scheduling, allocation, and binding of process [12].

\subsection{Problem Definition}

We are supposed to have different solution where we have two modules of solutions for defining the objective and principle of multiple supply-voltages scheduling problem that need to target two basic requirement. They may include consumption of peak power and also factors which affect other designing such as power in average, consumption of energy [12], and lastly area which may be depicted in the following Figure 4.

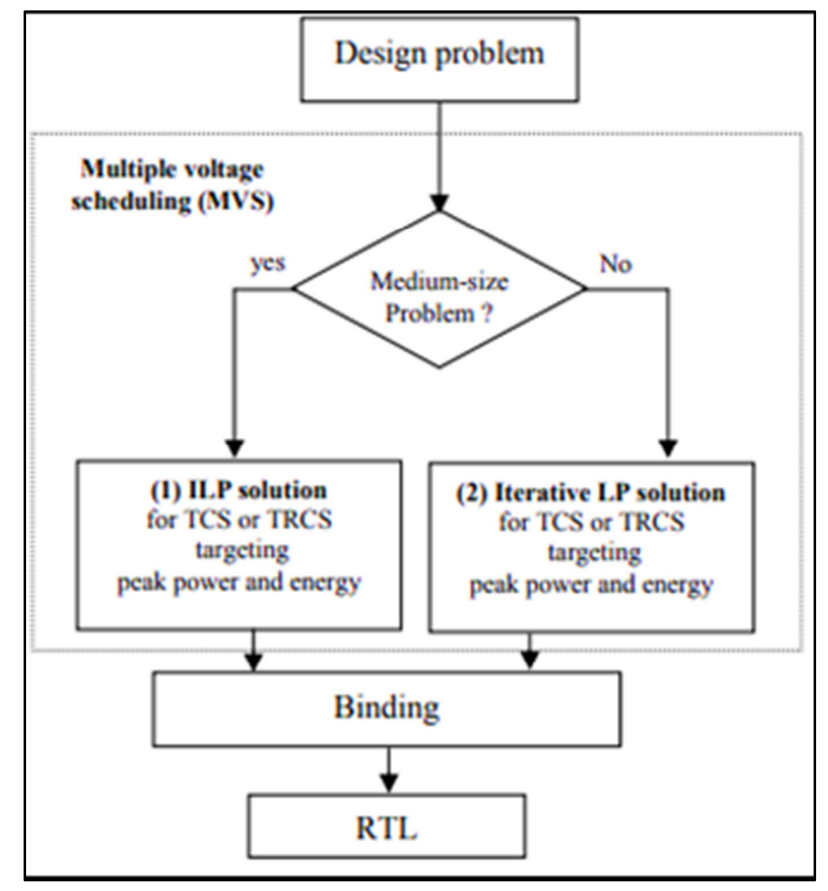

Figure 4. Flow-chart for Voltage Scheduling.

An optimal solution for problem which consists of medium 
range and approaches for large problems just to satisfies the large design of system. For designing the efficient space demand, the module for input library is supposed to be the modules which include delay values, area, and consumption of utility.

\subsection{Power-Area Saving}

The primary goal is to secure more power consumption and the saving area should have the flexibility any operation. Therefore the technique is to maintain and schedule any operation which have less intensive area of more power saving module or any operation which can have f]the flexibility of moving any desire task accordingly up or down within a range without getting violated from any power disturbance and thus saving.

\section{Conclusion}

Hence this paper reviewed the techniques and aims of analyzing different tools for power management and discussion of embedded system design methods works efficiently [13]. Thus the platform which consists of both hardware along with software and some optimization constraint techniques as well as strategies, the application is widely determine. Although there have been largest power to reduces gain that can be achieved with the help of voltage scaling, hence we have no work that tackled the power consumption for the process to minimize the control flow and have intensive applications throughout with this efficient technique.

\section{References}

[1] G. Nemhauser and L. Wolsey, Integer and Combinatorial Optimization, John Wiley, New York, 1988.

[2] P. G. Paulin and J. P. Knight, "Force Directed Scheduling for the Behavior Synthesis of ASICs," IEEE Transactions on CAD, vol. 8, pp. 661-679, June 1989.

[3] M. Potkonjak and J. M. Rabaey, "Optimizing Resource Utilization Using Transformations," IEEE Trans. on CAD of Integrated Circuits and Systems, vol. 13, no. 3, pp. 277-292, Mar. 1994.
[4] M. Rim, Y. Fann, and R. Jain, "Global Scheduling with Code-Motions for HighLevel Synthesis Applications," IEEE Trans. on VLSI Systems, vol. 3, pp. 379-392, Sep. 1995.

[5] F. Vahid and T. D. Givargis, Embedded system Design, A unified Hardware/Software Introduction. John Wiley and Sons, 2002.

[6] Z. Wakabayashi and T. Yoshimura, "A Resource Sharing and Control Synthesis Method for Conditional Branches," Proc. of International Conference on ComputerAided Design, pp. 62-65, Nov. 1989.

[7] F. Catthoor, F. Franssen, S. Wuytack, L. Nachtergaele, and H. De Man, "Global communication and memory optimizing transformations for low power signal processing systems," Proc. Int. Wkshp. on Low Power Design, pages 203-208, Apr. 1994.

[8] E. Chung, L. Benini and G. De Micheli, "Dynamic power management for non-stationary service requests", Proc. Design and Test in Europe Conference, pages 77-81, March 1999.

[9] P. Chandrakasan, M. Potkonjak, R. Mehra, J. Rabaey, and R. W. Broderson, "Optimizing power using transformations," IEEE Trans. Computer-Aided Design, Vol. 14, pages 12-51, Jan. 1995.

[10] L. Benini, G. DeMicheli, E. Macii, M. Poncino, and S. Quer, "System-level power optimization of special purpose applications: The beach solution," Proc. Symposium on Low Power Electronics and Design, pages 24-29, August 1997.

[11] C.-H. Hwang and A. Wu, "A predictive system shutdown method for energy saving of event-driven computation," Proc. Int.. Conf. on Computer Aided Design, pages 28-32, November 1997.

[12] J. L. da Silva, F. Catthoor, D. Verkest, and H. De Man, "Power exploration for dynamic data types through virtual memory management refinement," Proc. Int. Symp. Low Power Electronics and Design, Aug. 1999.

[13] V. Tiwari, S. Malik, and A. Wolfe, "Power analysis of embedded software: A first step towards software power minimization,” Proc. Int. Conf. Computer-Aided Design, Nov. 1994.

[14] H. Mehta, R. M. Owens, and M. J. Irwin, "Some issues in gray code addressing," Proc. Great Lakes Symposium on VLSI, Ames, IA, USA, pages 178-181, Mar. 1996.

[15] T. Pering, T. Burd, and R. Brodersen, "The simulation and evaluation of dynamic voltage scaling algorithms," Proc. Int. Symp. Low Power Electronics and Design, Aug. 1998. 